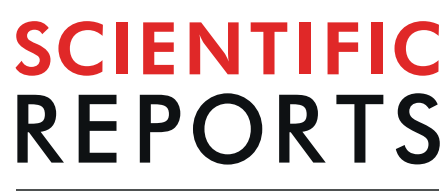

natureresearch

\title{
OPEN Connective tissue growth factor is correlated with peritoneal lymphangiogenesis
}

Received: 14 August 2018

Accepted: 7 August 2019

Published online: 21 August 2019

\author{
Hiroshi Kinashi ${ }^{1,2}$, Naohiro Toda ${ }^{3}$, Ting Sun ${ }^{4}$, Tri Q. Nguyen², Yasuhiro Suzuki ${ }^{4}$, \\ Takayuki Katsuno ${ }^{1}$, HidekiYokoi $\mathbb{1}^{3}$, Jan Aten ${ }^{5}$, Masashi Mizuno ${ }^{4}$, Shoichi Maruyama $\mathbb{D}^{4}$, \\ Motoko Yanagita $\mathbb{1}^{3}$, Roel Goldschmeding ${ }^{2}$ \& Yasuhiko Ito ${ }^{1}$
}

Lymphatic absorption in the peritoneal cavity may contribute to ultrafiltration failure in peritoneal dialysis (PD). Lymphatic vessels develop during PD-related peritoneal fibrosis. Connective tissue growth factor (CTGF, also called CCN2) is an important determinant of fibrotic tissue remodeling, but little is known about its possible involvement in lymphangiogenesis. In this study, we investigated the relationship between CTGF and peritoneal lymphangiogenesis. A positive correlation was observed between vascular endothelial growth factor-C (VEGF-C), a major lymphangiogenic growth factor, and the CTGF concentration in human PD effluents. CTGF expression was positively correlated with expression of lymphatic markers and VEGF-C in human peritoneal biopsies. We found a positive correlation between the increase in CTGF and the increase in VEGF-C in cultured human peritoneal mesothelial cells (HPMCs) treated with transforming growth factor- $\beta 1$ (TGF- $\beta 1$ ). The diaphragm is a central player in peritoneal lymphatic absorption. CTGF expression was also correlated with expression of VEGF-C and lymphatics in a rat diaphragmatic fibrosis model induced by chlorhexidine gluconate (CG). Furthermore, CTGF gene deletion reduced VEGF-C expression and peritoneal lymphangiogenesis in the mouse CG model. Inhibition of CTGF also reduced VEGF-C upregulation in HPMCs treated with TGF- $\beta 1$. Our results suggest a close relationship between CTGF and PD-associated lymphangiogenesis.

Peritoneal dialysis (PD) is one type of renal replacement therapy for patients with end-stage renal disease (ESRD). Dialysate in the peritoneal cavity removes waste products by diffusion through peritoneal capillaries. Excess fluids are also removed by osmotic agents such as hypertonic glucose in the dialysate. Water removal (or ultrafiltration) equals transcapillary water removal minus lymphatic absorption ${ }^{1}$ because peritoneal lymphatic vessels continuously drain intraperitoneal fluids and transport them to the circulation ${ }^{2}$.

Ultrafiltration failure (UFF) accompanied by high peritoneal solute transport is an important complication seen after long-term PD. UFF is a main reason for the discontinuation of PD treatment, and is also associated with poor survival of PD patients $\mathrm{s}^{3-6}$. The characteristic findings of chronic peritoneal damage in PD treatment are submesothelial fibrosis and neoangiogenesis ${ }^{7,8}$. However, the relationship between peritoneal fibrosis and UFF is still unclear. One potential mechanism focuses on lymphangiogenesis.

Clinical studies have shown that higher lymphatic absorption is associated with lower effective ultrafiltration $^{9}$ and $\mathrm{UFF}^{10}$. However, the results from these clinical approaches are controversial ${ }^{11,12}$, and the process of lymphangiogenesis remains poorly understood. We therefore investigated whether lymphangiogenesis is associated with peritoneal fibrosis ${ }^{13}$. Signaling via vascular endothelial growth factor (VEGF)-C/D and VEGF receptor (VEGFR)-3 is central to lymphangiogenesis ${ }^{14,15}$. During peritoneal fibrosis, transforming growth factor- $\beta$ (TGF- $\beta$ ) promotes VEGF-C expression, which leads to lymphangiogenesis ${ }^{13,16}$. Furthermore, blocking lymphangiogenesis with soluble VEGFR-3 improves impaired ultrafiltration in a mouse peritoneal fibrosis model ${ }^{17}$.

Connective tissue growth factor (CTGF) is a member of the CCN (CTGF/Cyr61/Nov) family and is an important player in the pathogenesis of fibrotic disorders. CTGF is increased in human PD effluents and human

\footnotetext{
${ }^{1}$ Department of Nephrology and Rheumatology, Aichi Medical University, Nagakute, Japan. ${ }^{2}$ Department of Pathology, University Medical Center Utrecht, Utrecht, The Netherlands. ${ }^{3}$ Department of Nephrology, Graduate School of Medicine, Kyoto University, Kyoto, Japan. ${ }^{4}$ Department of Nephrology and Renal Replacement Therapy, Nagoya University Graduate School of Medicine, Nagoya, Japan. ${ }^{5}$ Department of Pathology, Academic Medical Center, University of Amsterdam, Amsterdam, The Netherlands. Correspondence and requests for materials should be addressed toY.I. (email: yasuito@aichi-med-u.ac.jp)
} 


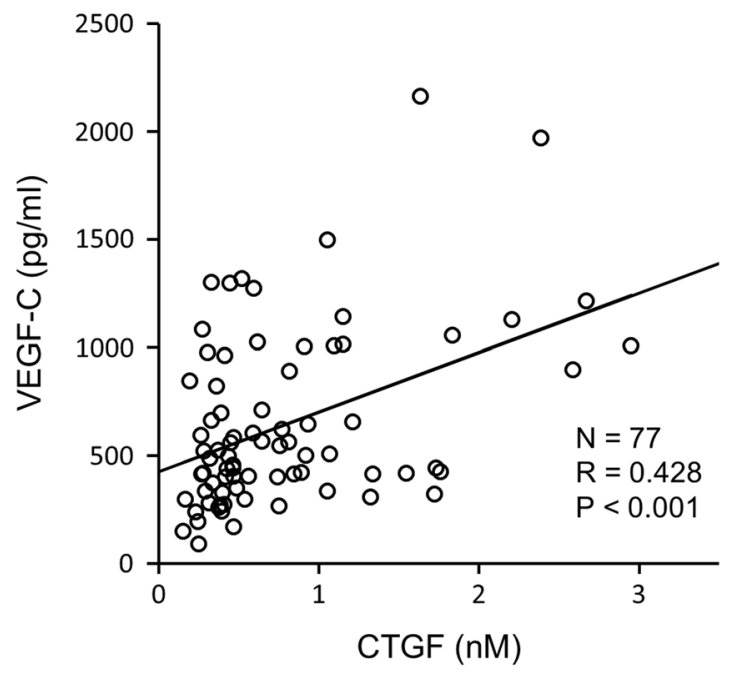

Figure 1. Positive correlation between connective tissue growth factor (CTGF) and vascular endothelial growth factor-C (VEGF-C) expression in human peritoneal dialysis (PD) effluents. CTGF and VEGF-C protein levels in 77 overnight dwelled human PD effluents were measured with a sandwich enzyme-linked immunosorbent assay. The CTGF concentration was positively correlated with the VEGF-C concentration in human PD effluents. Pearson correlation was used for the analysis.

peritoneal biopsy samples in association with a high peritoneal solute transport rate ${ }^{18}$. CTGF production by human peritoneal mesothelial cells (HPMCs) is regulated by advanced glycation end products, glucose degradation products, and TGF- $\beta^{18-20}$. In addition to the major regulatory role during fibrosis, CTGF is an important regulator of angiogenesis ${ }^{21}$. However, little is known about the possible association of CTGF with lymphangiogenesis.

We recently reported that CTGF promotes VEGF-C expression, which leads to fibrosis-related renal lymphangiogenesis ${ }^{22}$. The role of renal lymphangiogenesis seems to depend on the etiology of kidney diseases ${ }^{23-25}$. The role of peritoneal lymphatics in the physiology of PD is continuous absorption of intraperitoneal dialysate leading to a decrease in the ultrafiltration volume. Therefore, clarification of the mechanism of fibrosis-associated peritoneal lymphangiogenesis may lead to development of new therapeutic strategies for UFF.

In this study, we investigated the relationship between CTGF and VEGF-C expression in human PD effluents. We also explored the association of CTGF with VEGF-C and lymphatic vessels in human peritoneal biopsy samples. In vitro, we analyzed TGF- $\beta 1$-induced CTGF and VEGF-C upregulation in HPMCs collected from spent PD effluents. In addition, we analyzed lymphangiogenesis in the diaphragm, which we previously showed to play a central role in peritoneal lymphatic absorption ${ }^{13,17}$. This was done in the chlorhexidine gluconate (CG)-induced rat peritoneal fibrosis model ${ }^{13,26}$. To explore the role of CTGF in peritoneal lymphangiogenesis, we assessed the effect of CTGF reduction on peritoneal lymphangiogenesis using CTGF conditional knockout $\left(\mathrm{CTGF}^{-1-}\right)$ mice $^{27,28}$. We also investigated the effect of CTGF inhibition on TGF- $\beta 1$-induced VEGF-C upregulation in HPMCs using CTGF small interfering RNA (siRNA).

\section{Results}

Positive correlation between CTGF and VEGF-C expression in human PD effluents. To explore the involvement of CTGF in peritoneal lymphangiogenesis, we measured the CTGF and VEGF-C concentration in the overnight dwelled PD effluents derived from 77 patients. The mean CTGF concentration was $0.79 \pm 0.63$ (range $=0.16-2.95) \mathrm{nM}$. The mean VEGF-C concentration was $643 \pm 407$ (range $=89.3-2161) \mathrm{pg} / \mathrm{ml}$. A positive correlation was observed between the CTGF and VEGF-C concentration in the PD effluents $(\mathrm{R}=0.428$, $\mathrm{P}<0.001$, Fig. 1).

CTGF expression was correlated with expression of lymphatics and VEGF-C in human peritoneal biopsy samples. Second, we analyzed the messenger RNA (mRNA) expression of CTGF, VEGF-C, and lymphatic markers (lymphatic vessel endothelial hyaluronan receptor-1 (LYVE-1) and podoplanin) in 62 human peritoneal biopsy samples derived from pre-dialysis uremic patients $(n=32)$ and PD patients with or without UFF ( $\mathrm{n}=7, \mathrm{n}=23$, respectively). CTGF mRNA expression was positively correlated with LYVE-1 $(\mathrm{R}=0.698$, $\mathrm{P}<0.001$, Fig. $2 \mathrm{a})$, podoplanin $(\mathrm{R}=0.666, \mathrm{P}<0.001$, Fig. $2 \mathrm{~b})$, and VEGF-C $(\mathrm{R}=0.727, \mathrm{P}<0.001$, Fig. $2 \mathrm{c}) \mathrm{mRNA}$ expression in the human peritoneal membranes.

We also analyzed the correlations between the mRNAs in each category. CTGF mRNA expression was similarly correlated with LYVE-1 $(\mathrm{R}=0.571, \mathrm{P}<0.01 ; \mathrm{R}=0.476, \mathrm{P}<0.05$, Supplementary Figs 1a and 2a), podopla$\operatorname{nin}(\mathrm{R}=0.577, \mathrm{P}<0.01 ; \mathrm{R}=0.500, \mathrm{P}<0.05$, Supplementary Figs $1 \mathrm{~b}$ and $2 \mathrm{~b})$, and VEGF- $\mathrm{C}(\mathrm{R}=0.713, \mathrm{P}<0.001$; $\mathrm{R}=0.478, \mathrm{P}<0.05$, Supplementary Figs $1 \mathrm{c}$ and $2 \mathrm{c}$ ) mRNA expression in the peritoneum of pre-PD patients and in the peritoneum of PD patients without UFF, respectively. CTGF mRNA expression tended to be correlated with LYVE-1 and podoplanin mRNA expression in the peritoneum of PD patients with UFF, but the relationship was not statistically significant (Supplementary Fig. 3a,b). However, CTGF mRNA expression was significantly correlated with VEGF-C mRNA expression in the UFF peritoneum $(\mathrm{R}=0.781, \mathrm{P}<0.05$, Supplementary Fig. $3 \mathrm{c})$. 
a

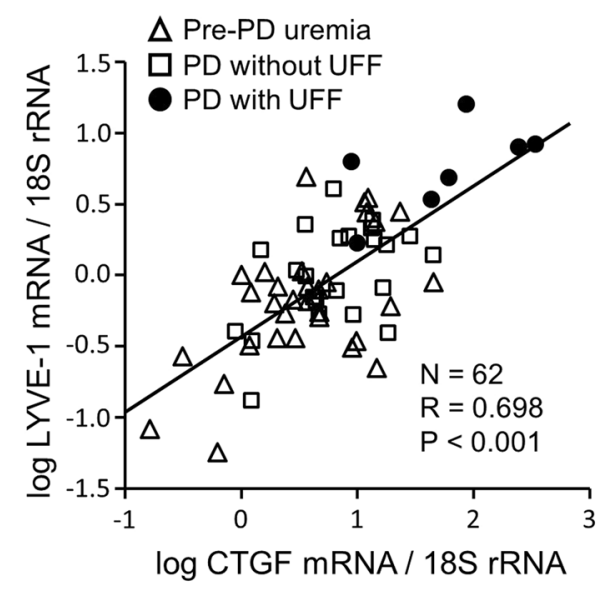

C

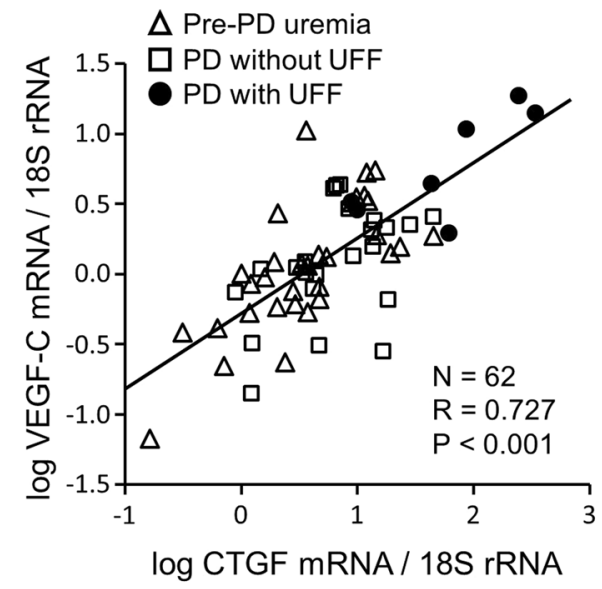

b

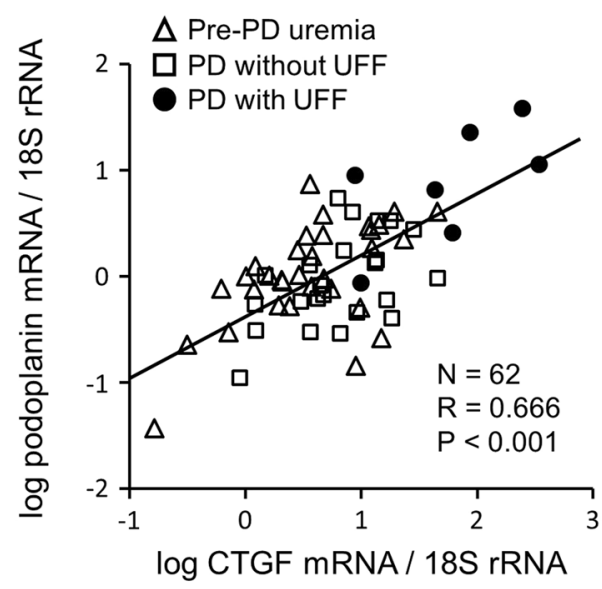

Figure 2. Connective tissue growth factor (CTGF) expression was correlated with expression of lymphatic markers and vascular endothelial growth factor-C (VEGF-C) in human peritoneal biopsies. Human peritoneal biopsy specimens were collected from 32 uremic patients before initiation of peritoneal dialysis (PD), 23 patients undergoing PD not complicated by ultrafiltration failure (UFF), and seven PD patients with UFF. Quantitative polymerase chain reaction analysis showed positive correlations between CTGF messenger RNA (mRNA) expression and lymphatic vessel endothelial hyaluronan receptor-1 (LYVE-1) (a), podoplanin (b), and VEGF-C (c) mRNA expression in 62 human peritoneal biopsy samples. $18 \mathrm{~S}$ ribosomal RNA (rRNA) was used as an internal reference. Values were transformed into the logarithmic scale for Pearson correlation.

In addition, we investigated representative human peritoneal biopsies derived from a pre-PD patient and a PD patient with UFF by performing immunohistochemistry (IHC) for CTGF, VEGF-C, and a lymphatic marker (D2-40). Serial sections of the UFF peritoneum showed more D2-40-positive lymphatic vessels accompanied by increased expression of CTGF and VEGF-C than the pre-PD peritoneum (Supplementary Fig. 4).

The increase in CTGF mRNA expression was correlated with the increase in VEGF-C mRNA expression in HPMCs treated with TGF- $\beta 1$. Next, we assessed CTGF and VEGF-C mRNA expression in HPMCs derived from $21 \mathrm{PD}$ patients with different peritoneal membrane transport functions. Cells were cultured with or without TGF- $\beta 1(5 \mathrm{ng} / \mathrm{ml})$ and harvested $12 \mathrm{~h}$ later. CTGF and VEGF-C mRNA expression was increased to varying degrees by TGF- $\beta 1$ treatment. A positive correlation was observed between the fold-increase in CTGF and the fold-increase in VEGF-C mRNA $(\mathrm{R}=0.722, \mathrm{P}<0.001$, Fig. 3$)$ after TGF- $\beta 1$ treatment.

CTGF expression was correlated with expression of VEGF-C and lymphatic vessels in the rat CG-induced diaphragmatic fibrosis model. IHC analysis showed that expression of CTGF $(\mathrm{P}<0.01)$, VEGF-C $(\mathrm{P}<0.01)$, and LYVE-1-positive lymphatic vessels $(\mathrm{P}<0.01)$ was increased in the diaphragm in rats intraperitoneally injected with CG $(n=9)$ compared with control rats treated with saline $(n=5)$ (Fig. 4a,b, Supplementary Fig. 5). In addition, increased expression of CTGF mRNA was observed in the CG model with in situ hybridization (ISH) (Fig. 4a). Quantitative polymerase chain reaction (qPCR) analysis showed that mRNA expression of CTGF, VEGF-C, LYVE-1, and podoplanin was increased 4.8- $(\mathrm{P}<0.01), 2.2-(\mathrm{P}<0.01)$, 2.3- $(\mathrm{P}<0.01)$, and 3.3-fold $(\mathrm{P}<0.01)$, respectively, in the diaphragm in CG-injected rats compared to controls (Fig. 4c). Quantification of IHC showed that CTGF expression was positively correlated with expression 


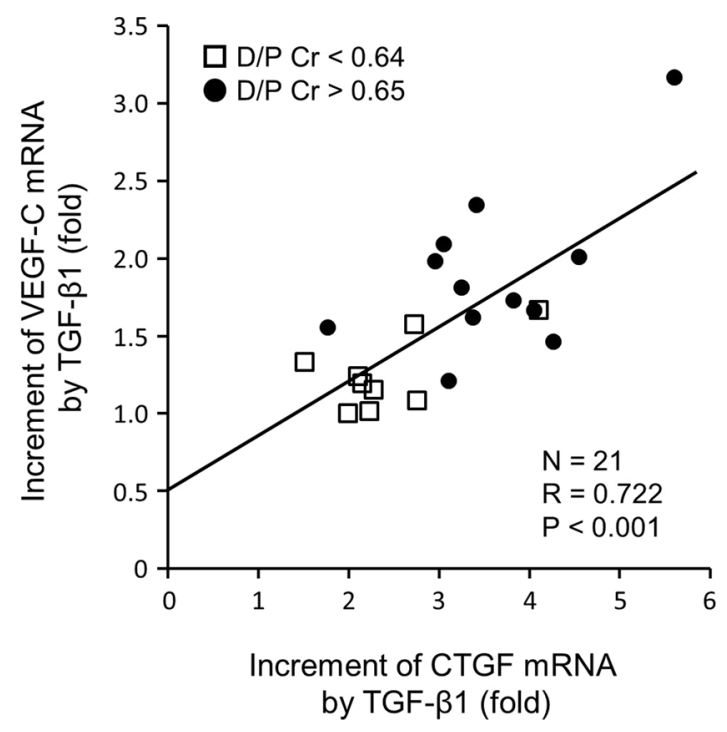

Figure 3. Positive correlation between the increase in connective tissue growth factor (CTGF) and the increase in vascular endothelial growth factor-C (VEGF-C) in human peritoneal mesothelial cells (HPMCs) treated with transforming growth factor- $\beta 1$ (TGF- $\beta 1$ ). HPMCs were collected from the spent peritoneal dialysis (PD) effluents derived from 21 PD patients with various peritoneal transport functions. Nine patients showed lower peritoneal permeability (dialysate to plasma ratio of creatinine, $\mathrm{D} / \mathrm{P} \mathrm{Cr}<0.64$ ), and the other 12 patients showed higher peritoneal permeability $(\mathrm{D} / \mathrm{P} \mathrm{Cr}>0.65)$. Cultured HPMCs were treated with $5 \mathrm{ng} / \mathrm{ml}$ recombinant human TGF- $\beta 1$ for $12 \mathrm{~h}$ and harvested. Increases in CTGF and VEGF-C messenger RNA (mRNA) following incubation with TGF- $\beta 1$ were analyzed with quantitative polymerase chain reaction. A positive correlation was observed between CTGF and VEGF-C mRNA amplification in HPMCs treated with TGF- $\beta 1$. $18 \mathrm{~S}$ ribosomal RNA (rRNA) was used as an internal reference. Pearson correlation was used for the analysis.

of VEGF-C ( $\mathrm{R}=0.952, \mathrm{P}<0.001$, Fig. $4 \mathrm{~d})$, and LYVE-1-positive lymphatic vessels $(\mathrm{R}=0.775, \mathrm{P}<0.05$, Fig. 4e) in the rats injected with CG. Moreover, a positive correlation was observed between VEGF-C and LYVE-1 expression in these rats $(\mathrm{R}=0.704, \mathrm{P}<0.05$, Fig. $4 \mathrm{f})$. Additionally, double immunofluorescent staining of the CG-treated diaphragm showed more LYVE-1-positive lymphatic vessels in regions with increased expression of CTGF than in the control diaphragm (Fig. 4g).

Genetic deletion of CTGF reduced lymphangiogenesis and VEGF-C expression in the mouse CG-induced peritoneal fibrosis model. We investigated the effect of genetic deletion of CTGF on CG-induced peritoneal lymphangiogenesis using tamoxifen-inducible conditional $\mathrm{CTGF}^{-1-}$ mice. IHC of the parietal peritoneum showed that LYVE-1-positive lymphatic vessels and VEGF-C expression were barely present in the phosphate-buffered saline (PBS)-treated peritoneum (Fig. 5a). In contrast, dilated lymphatic vessels appeared and VEGF-C expression was increased in CG-induced peritoneal fibrosis (Fig. 5a). IHC analysis showed that the LYVE-1-positive area and VEGF-C score (Supplementary Fig. 6) were significantly increased in the CG-treated control peritoneum compared with the PBS-treated control peritoneum $(\mathrm{P}<0.001, \mathrm{P}<0.05$, respectively, Fig. 5b,c), and were significantly decreased in the CG-treated $\mathrm{CTGF}^{-/-}$peritoneum compared with the CG-treated control peritoneum $(\mathrm{P}<0.05$, Fig. 5b,c). We found no significant differences in the LYVE1-positive area and VEGF-C score between the PBS-treated control peritoneum and PBS-treated CTGF ${ }^{-1-}$ per- $^{-}$ itoneum (Fig. 5b,c). qPCR analysis showed that VEGF-C and VEGFR-3 mRNA were increased 4.9- $(\mathrm{P}<0.001)$ and 12.7-fold $(\mathrm{P}<0.001)$, respectively, in the CG-treated control peritoneum compared with the PBS-treated control peritoneum. The CG-treated $\mathrm{CTGF}^{-1-}$ peritoneum showed a significant decrease in both VEGF-C and VEGFR-3 mRNA compared with the CG-treated control peritoneum $(\mathrm{P}<0.01, \mathrm{P}<0.001$, respectively, Fig. $5 \mathrm{~d}, \mathrm{e})$. We found no significant differences in VEGF-C or VEGFR-3 mRNA expression between the PBS-treated control peritoneum and the PBS-treated $\mathrm{CTGF}^{-1-}$ peritoneum (Fig. 5d,e). Double immunofluorescent staining showed that the expression pattern of VEGFR-3-positive lymphatic vessels was similar to the expression pattern of LYVE-1-positive lymphatic vessels in the CG-treated control peritoneum and CG-treated CTGF ${ }^{-1-}$ peritoneum (Supplementary Fig. 7).

siRNA-mediated knockdown of CTGF reduced VEGF-C expression in HPMCs treated with TGF- $\beta 1$. Finally, we assessed the effect of siRNA-mediated knockdown of CTGF on VEGF-C expression in HPMCs. We inhibited CTGF expression with CTGF siRNA in HPMCs treated with TGF- 31 . CTGF and VEGF-C mRNA expression was increased 2.7- $(\mathrm{P}<0.001)$ and 2.4-fold $(\mathrm{P}<0.001)$, respectively, in HPMCs after $24 \mathrm{~h}$ of incubation with TGF- $\beta 1$ (Fig. 6a,b). CTGF siRNA significantly reduced TGF- $\beta 1$-stimulated CTGF and VEGF-C expression ( $\mathrm{P}<0.001, \mathrm{P}<0.01$, respectively) compared with negative control siRNA (Fig. 6a,b). 


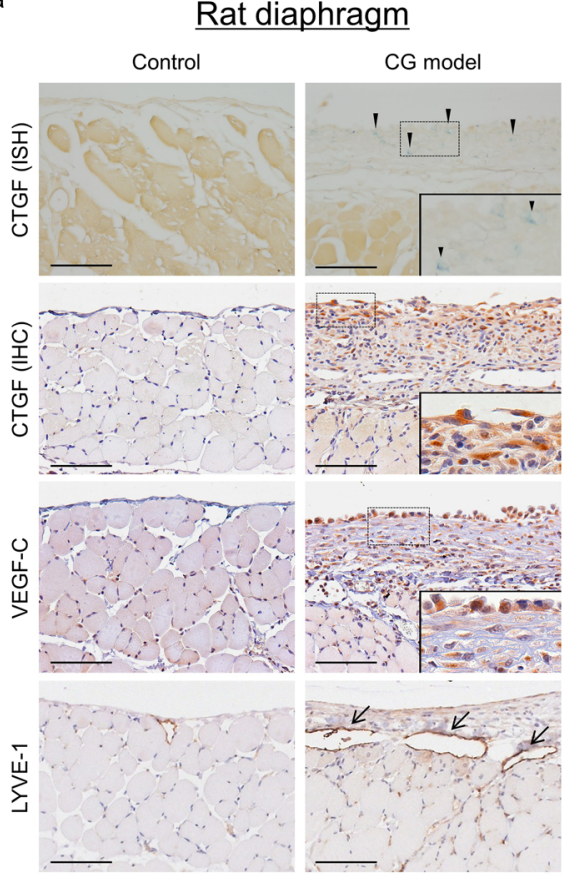

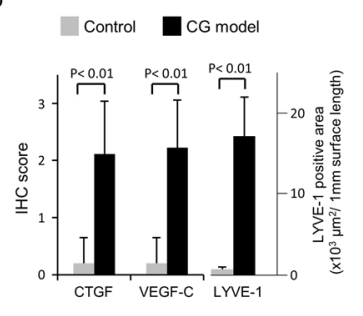

d

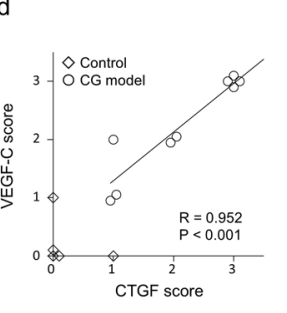

e
C

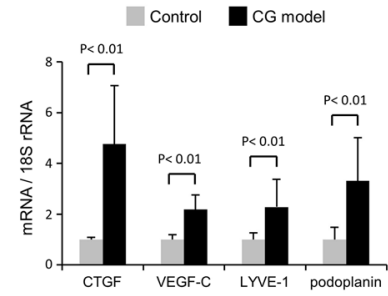

$\mathrm{f}$

g
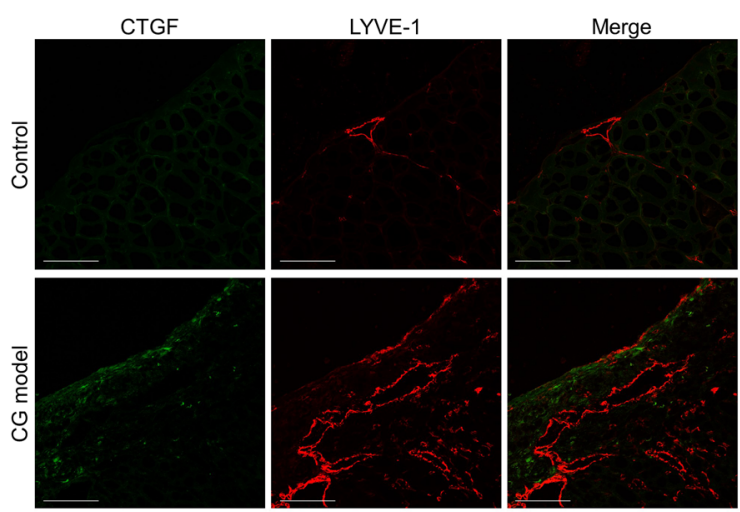

Figure 4. Connective tissue growth factor (CTGF) expression was correlated with expression of vascular endothelial growth factor-C (VEGF-C) and lymphatics in a rat diaphragmatic fibrosis model induced by chlorhexidine gluconate (CG). Diaphragmatic fibrosis was induced by intraperitoneal injection of CG in rats. Control rats were treated with saline. (a,b) Immunohistochemistry (IHC) showed that expression of CTGF, VEGF-C, and lymphatic vessel endothelial hyaluronan receptor-1 (LYVE-1) was increased in the rat diaphragmatic fibrosis model induced by CG compared with controls. The staining intensity for CTGF and VEGF-C was scored as follows: 0, absent; 1, mild; 2, moderate; 3, extensive. CTGF messenger RNA (mRNA) was detected in the CG model with in situ hybridization (ISH). Arrowheads indicate CTGF mRNA expression. Arrows indicate LYVE-1-positive lymphatic vessels. Insets show magnification of the dotted-line boxed areas. Scale bars; $100 \mu \mathrm{m}$. (c) CTGF, VEGF-C, LYVE-1, and podoplanin mRNA expression were increased in the diaphragm in CG-injected rats compared with controls as seen with quantitative polymerase chain reaction analysis. (d-f) IHC analysis showed positive correlations among CTGF, VEGF-C, and LYVE-1 expression in the CG model. Spearman's rank correlation was used for the analysis. (g) Double immunofluorescent staining showed that CTGF expression was increased around LYVE-1-positive lymphatic vessels in the CG model. Bar graphs show means $\pm \mathrm{SD}$ (Control, $\mathrm{n}=5$; CG model, $\mathrm{n}=9$ ).

\section{Discussion}

Involvement of lymphatic absorption in the physiology of PD was noticed three decades ago ${ }^{1}$. Clinical studies showed that higher lymphatic absorption is associated with PD duration and UFF ${ }^{9,29}$. However, the effective lymphatic absorption rate, which was estimated by the disappearance of intraperitoneally administered macromolecules, such as radioactive iodinated serum albumin or dextran, is controversial ${ }^{11,12}$. Lymphatics in PD have been less well studied in the recent decade. In contrast, owing to the recent discovery of several useful lymphatic markers and key molecular mechanisms, lymphangiogenesis has been studied in a variety of diseases, such as tumor metastasis $^{30}$, inflammatory disease ${ }^{31}$, heart disease ${ }^{32}$, and transplant rejection ${ }^{33}$. We observed lymphangiogenesis in various types of human kidney diseases, and demonstrated that the number of lymphatic vessels is correlated with the degree of renal interstitial fibrosis ${ }^{34}$. Lymphangiogenesis is also associated with human peritoneal fibrosis, and patients with UFF show high expression of lymphatic vessel markers in the peritoneum compared to PD patients without $\mathrm{UFF}^{13}$. Interestingly, investigations using the rat remnant kidney model recently revealed that chronic kidney disease itself can induce peritoneal fibrosis, lymphangiogenesis, and a high lymphatic absorption rate, independent of exposure to PD solution ${ }^{35}$. Two major mechanisms, i.e., neoangiogenesis accompanied by high vascular permeability, and reduction of the osmotic gradient by tissue fibrosis, are highlighted in the context 

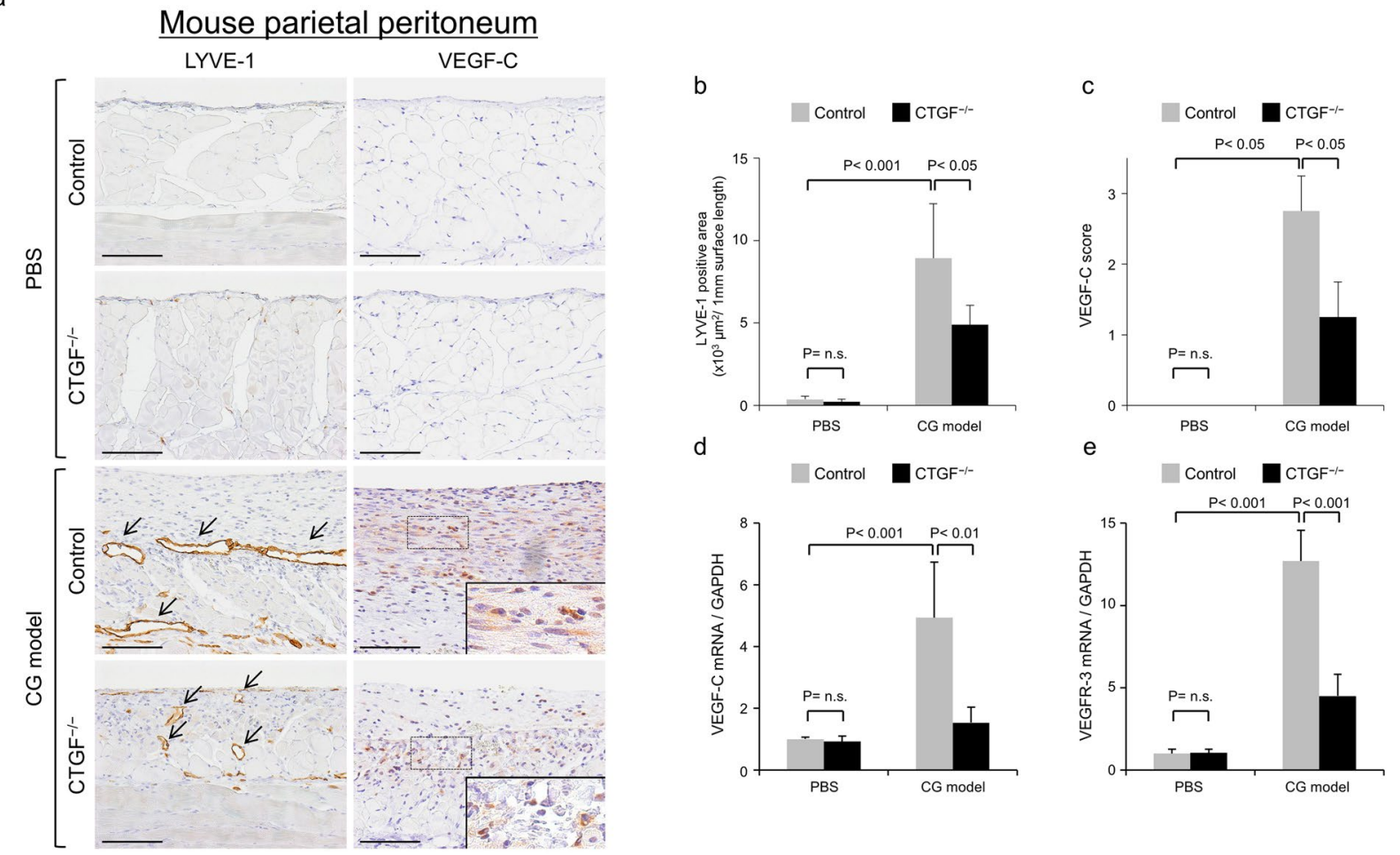

Figure 5. Genetic deletion of connective tissue growth factor (CTGF) reduced lymphangiogenesis and vascular endothelial growth factor-C (VEGF-C) expression in a mouse peritoneal fibrosis model induced by chlorhexidine gluconate (CG). Peritoneal fibrosis was induced by intraperitoneal injection of CG in wildtype mice (Control) and CTGF knockout $\left(\mathrm{CTGF}^{-1-}\right)$ mice. PBS-treated mice were used for comparison. $(\mathbf{a}-\mathbf{c})$ Immunohistochemical analysis showed that the increased expression of lymphatic vessel endothelial hyaluronan receptor-1 (LYVE-1) and VEGF-C in the CG model was significantly decreased in $\mathrm{CTGF}^{-1-}$ mice compared with control mice. We found no significant differences in LYVE-1 and VEGF-C expression between PBS-treated control peritoneum and PBS-treated $\mathrm{CTGF}^{-1-}$ peritoneum. The staining intensity for VEGF-C was scored as follows: 0, absent; 1, mild; 2, moderate; 3, extensive. Arrows indicate LYVE-1-positive lymphatic vessels. Insets show magnification of dotted-line boxed areas. Scale bars; $100 \mu \mathrm{m}$. (d,e) Quantitative polymerase chain reaction analysis showed that the increased expression of VEGF-C and VEGF receptor-3 (VEGFR-3) messenger RNA (mRNA) in the CG model was significantly decreased in $\mathrm{CTGF}^{-1-}$ mice compared with control mice. We found no significant differences in VEGF-C or VEGFR-3 mRNA expression between the PBS-treated control peritoneum and PBS-treated $\mathrm{CTGF}^{-1-}$ peritoneum. Glyceraldehyde-3-phosphate dehydrogenase (GAPDH) was used as an internal reference. Graphs show means $\pm S D(n=4$ for each group). n.s.; not significant.

of UFF in peritoneal fibrosis ${ }^{36}$. However, fibrosis-associated lymphangiogenesis additionally suggests the possible involvement of lymphatic absorption in the UFF mechanism of progressive peritoneal membrane injury.

In this study, we first explored whether the CTGF concentration was positively correlated with the VEGF-C concentration in human PD effluents. Both CTGF and VEGF-C protein in human dialysate are correlated with peritoneal permeability ${ }^{13,18,37}$, suggesting that both could be biomarkers and therapeutic targets for UFF. The dialysate protein level is affected by local peritoneal production and transfer from the blood circulation, which is also dependent on several factors including the plasma level ${ }^{38}$, peritoneal protein clearance ${ }^{39}$, molecular weight with degradation ${ }^{18}$, and protein charge ${ }^{40}$. Although the data include complicated factors, our findings are compatible with a role for CTGF in lymphangiogenesis in the peritoneum.

CTGF was detected and present at higher levels in mesothelial cells and fibroblasts in human fibrotic peritoneal membranes in relation to UFF ${ }^{18}$. mRNA expression of CTGF, VEGF-C, and lymphatic markers in peritoneum is higher in UFF patients than in pre-dialysis uremia patients, and is correlated with peritoneal thickness $^{13,18}$. Interestingly, no correlation is present between CTGF mRNA expression and the density of blood vessels in human peritoneal biopsies ${ }^{18}$. Unlike the blood vessel analysis, however, our current study showed that CTGF mRNA expression was positively correlated with VEGF-C, LYVE-1, and podoplanin mRNA expression in human peritoneal biopsies. Our results indicate that local CTGF expression in the context of peritoneal fibrosis is closely linked to lymphangiogenesis.

Mesothelial cells that line the surface of the peritoneal cavity predominantly regulate intraperitoneal homeostasis including the synthesis of cytokines, growth factors, and matrix proteins ${ }^{41}$. In parallel with the enhancement of profibrotic activity by CTGF production, mesothelial cells also play an important role in lymphangiogenesis 
a

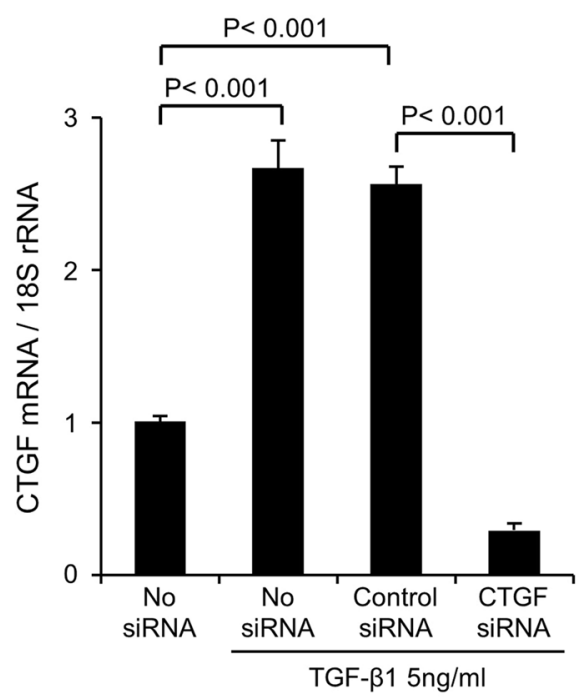

b

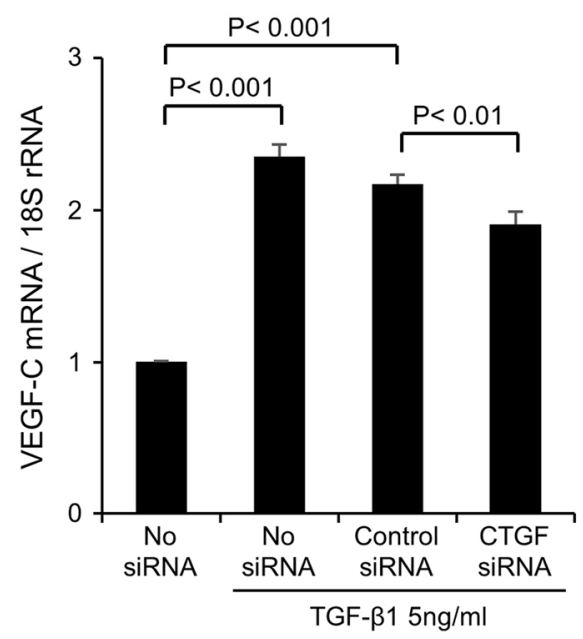

Figure 6. Small interfering RNA (siRNA)-mediated knockdown of connective tissue growth factor (CTGF) reduced vascular endothelial growth factor-C (VEGF-C) upregulation in human peritoneal mesothelial cells (HPMCs) treated with transforming growth factor- $\beta 1$ (TGF- $\beta 1$ ). HPMCs were collected from the peritoneal dialysis (PD) effluent derived from a PD patient with high peritoneal permeability. CTGF messenger RNA (mRNA) expression was inhibited by siRNA in cultured HPMCs treated with $5 \mathrm{ng} / \mathrm{ml}$ recombinant human TGF- $\beta 1$ for $24 \mathrm{~h}$. Quantitative polymerase chain reaction analysis showed that the TGF- $\beta 1$-induced mRNA upregulation of both CTGF and VEGF-C was reduced by CTGF siRNA compared with control siRNA. 18S ribosomal RNA (rRNA) was used as an internal reference. Graphs show means \pm SD ( $n=3$ for each group).

by producing VEGF-C. We treated HPMCs from spent PD effluent with TGF- $\beta 1$, which can be elevated in the peritoneal cavity by several mechanisms, such as exposure to dialysate glucose $\mathrm{e}^{42}$, advanced glycation end products $^{43}$, glucose degradation products ${ }^{20}$, and the presence of bacterial peritonitis ${ }^{44}$. Our results demonstrated that production of both CTGF and VEGF-C was increased by TGF- $\beta 1$ to varying degrees in HPMCs from individual patients, and that their enhancement showed a positive correlation. This is in accordance with the findings in PD effluent and peritoneal biopsy analyses.

The diaphragm contains a specialized form of the lymphatic absorption system including lymphatic lacunae and mesothelial stomata ${ }^{2}$. This aspect of UFF and lymphangiogenesis can be studied only in animal experiments. IHC of diaphragm sections from the rat CG model showed that CTGF is mainly increased in peritoneal mesothelial cells and fibroblast-like cells, and that VEGF-C is increased in mesothelial cells and mononuclear infiltrates, consistent with observations in the parietal peritoneum ${ }^{13,18}$. CTGF expression in the diaphragm is significantly correlated with expression of VEGF-C and LYVE-1-positive lymphatic vessels, supporting the concept of the involvement of CTGF in diaphragmatic lymphangiogenesis.

Several animal experiments demonstrated that CTGF inhibition ameliorates the development of fibrosis in obstructive nephropathy ${ }^{45}$, diabetic nephropathy ${ }^{46}$, allograft nephropathy ${ }^{47}$, and the remnant kidney model ${ }^{48}$. In addition, recent studies showed that CTGF inhibition ameliorates CG-induced peritoneal fibrosis through suppression of fibroblast accumulation, angiogenesis, and inflammation ${ }^{28,49}$. Furthermore, we recently reported that CTGF is involved in fibrosis-associated renal lymphangiogenesis through regulation of, and direct interaction with, VEGF-C ${ }^{22}$. CTGF knockout results in suppression of lymphangiogenesis in obstructive nephropathy and an ischemia reperfusion injury model, which is congruent with the effect of CTGF inhibition on peritoneal lymphangiogenesis in this study.

CTGF directly binds to VEGF-C and inhibits VEGF-C-induced growth of lymphatic endothelial cells ${ }^{22}$. Therefore, we suggest that the reduction in lymphangiogenesis in the CG-treated $\mathrm{CTGF}^{-1-}$ peritoneum was mediated by not the absence of CTGF but by decreased VEGF-C expression. However, the effect of CTGF inhibition on VEGF-C reduction was smaller in vitro than in vivo. Regarding this point, we noticed that the CG-treated $\mathrm{CTGF}^{-1-}$ peritoneum showed less TGF- $\beta 1$ mRNA expression than the CG-treated control peritoneum $^{28}$. TGF- $\beta 1$ promotes VEGF-C expression in peritoneal mesothelial cells and macrophages as shown in our previous reports ${ }^{13,50}$. We suggest that the reduction in lymphangiogenesis in the CG-treated $\mathrm{CTGF}^{-l-}$ peritoneum is due to decreased TGF- $\beta$ levels in addition to less VEGF-C induction by TGF- $\beta$ due to little CTGF expression.

The specific blocking of VEGFR-3 signals using soluble VEGFR-3, a decoy receptor for VEGF-C and VEGF-D, significantly reduces lymphangiogenesis without altering inflammation or fibrosis in a mouse peritoneal fibrosis model induced by methylglyoxal, a toxic glucose degradation product ${ }^{17}$. In contrast, CTGF inhibition significantly reduces peritoneal fibrosis and inflammation in the mouse CG model ${ }^{28}$. Therefore, we propose that blocking VEGFR-3 signals specifically suppresses lymphangiogenesis in peritoneal fibrosis, which is different from the effect of CTGF inhibition. 
In conclusion, we have identified a close association between CTGF expression and lymphangiogenesis in peritoneal fibrosis. Studies are underway to clarify the possible benefit of targeting CTGF to prevent lymphangiogenesis, UFF, and peritoneal fibrosis in the course of PD therapy.

\section{Methods}

Patient profiles. The studies with human samples were performed in accordance with the ethical guidelines of the 1975 Declaration of Helsinki and were approved by the Ethics Committee for Human Research of the Faculty of Medicine, Nagoya University (approval no. 298: peritoneal fluid experiment; approval no. 299: peritoneal tissue experiments). Informed consent was obtained from all patients.

CTGF and VEGF-C protein levels were measured in 77 overnight dwelled $(8.97 \pm 1.62 \mathrm{~h})$ human peritoneal effluent samples ( 24 women and 53 men), which were collected at Nagoya University Hospital (Nagoya, Japan) and affiliated hospitals between July 2005 and April $2008^{13,18}$. The mean age of all patients was $54.7 \pm 13.0$ (range $=28-84$ ) years, and the mean period of PD treatment was $32.1 \pm 32.6$ (range $=1-132$ ) months. Diabetic nephropathy was the cause of ESRD in 26 PD patients (33.8\%). No patients had the complication of peritonitis for over 1 month before the study, and patients with other diseases, such as liver or lung diseases and malignancy, were not included. Patients treated with combination therapy (hemodialysis + PD) were excluded from this study. Peritoneal transport was calculated by the dialysate-to-plasma ratio of creatinine (D/P Cr), and the average value was $0.68 \pm 0.13$ (range $=0.28-0.96$ ).

A total of 62 peritoneal tissue samples were collected from 32 pre-dialysis chronic kidney disease patients at the time of PD catheter insertion and 30 PD patients at the time of PD catheter removal for PD-related complications. The mean age of pre-dialysis uremia patients (nine women and 23 men) was $61.3 \pm 12.4$ years. Among 30 PD patients, seven patients (four women and three men) were regarded as having UFF and needed to be treated with more than four hypertonic bags (2.27\% glucose and $3.86 \%$ glucose or icodextrin) per day to maintain their fluid status ${ }^{51}$. The mean age of UFF patients was $55.9 \pm 11.6$ years, and the mean period of PD treatment was $9.4 \pm 6.6$ years. The other $23 \mathrm{PD}$ patients ( 10 women and $13 \mathrm{men}$ ) had their catheters removed for reasons other than UFF, such as transplantation, mental disorders, severe exit site infection, or difficulty with performing bag exchanges. The mean age of the 23 patients without UFF was $60.8 \pm 13.2$ years, and the mean period of PD treatment was $3.7 \pm 3.0$ years. Correlations between CTGF and LYVE-1, podoplanin, and VEGF-C mRNA expression were evaluated ${ }^{13,18}$.

Cell culture study. HPMCs were collected by centrifugation of PD effluents derived from 21 clinically stable patients using modified methods as described previously ${ }^{13,18}$. Nine patients (four women and five men) were classified into the low or low average category of the peritoneal equilibration test $(\mathrm{D} / \mathrm{P} \mathrm{Cr}<0.64)$, and the other 12 patients (six women and six men) were classified into the high or high average category ( $/ \mathrm{P} \mathrm{Cr}>0.65)$. Subconfluent cells plated in $6-\mathrm{cm}$ dishes were rinsed with PBS, and the cultures were replaced with serum-free medium for $24 \mathrm{~h}$ for a quiescent state. Thereafter the cultures were incubated with or without $5 \mathrm{ng} / \mathrm{ml}$ recombinant human TGF- $\beta 1$ (240-B, R\&D Systems, Minneapolis, MN), which was mixed in serum-free medium. Cultures were harvested at $12 \mathrm{~h}(\mathrm{n}=3)$. All experiments were performed during the third to fourth passage. To explore the correlation between CTGF and VEGF-C upregulation by TGF- $\beta 1$, we assessed the fold-increase in CTGF and VEGF-C mRNA treated with TGF- $\beta 1$ compared with the basal mRNA expression without TGF- $\beta 1$ treatment.

For the CTGF inhibition study, HPMCs derived from a patient with high peritoneal permeability were used. Cells were transfected with $10 \mathrm{nM}$ silencer select siRNA for CTGF or a negative control (Ambion, Austin, TX) using Lipofectamine RNAiMAX (Invitrogen, Carlsbad, CA). After $24 \mathrm{~h}$ of transfection, culture medium was replaced with serum-free medium alone or medium with $5 \mathrm{ng} / \mathrm{ml}$ TGF- $\beta 1$. Cells were harvested after $24 \mathrm{~h}$ of incubation $(\mathrm{n}=3)$.

Animal models. The rat study was performed in accordance with the Animal Experimentation Guidelines of Nagoya University Graduate School of Medicine (Nagoya, Japan), and was approved by the Animal Experimentation Committee of Nagoya University (approval no. 23325). Eight-week-old male Sprague-Dawley rats (Japan SLC, Hamamatsu, Japan) were used for the study. Nine rats were intraperitoneally injected every other day with $3 \mathrm{ml} / 200 \mathrm{~g}$ body weight of $0.04 \%$ CG (Wako, Japan) and $10 \%$ ethanol (Wako) dissolved in saline ${ }^{13}$. Five control rats were treated with the same dose of saline. All diaphragm samples were collected on day 16.

The mouse experiment was performed according to the guidelines of the Animal Experimentation Committee of Kyoto University (Kyoto, Japan), and was approved by the Animal Experimentation Committee of Kyoto University (approval no. MedKyo17174, MedKyo16526, MedKyo15250). ROSA26-ERT2CRE/floxCTGF mice were treated with tamoxifen to establish $\mathrm{CTGF}^{-1-}$ mice as previously described ${ }^{27,28}$. Mice were intraperitoneally injected three times a week with $0.3 \mathrm{ml}$ of $0.1 \%$ CG in $15 \%$ ethanol dissolved in $\mathrm{PBS}^{28,52}$. The other mice were injected with the same dose of PBS for comparison. CG injection induces peritoneal fibrosis, inflammation, and neoangiogenesis ${ }^{28}$. All mice were euthanized after 4 weeks, and parietal peritoneal samples were collected $(n=4)$.

The harvested animal samples were analyzed with IHC, ISH, and qPCR.

Enzyme-linked immunosorbent assay (ELISA). CTGF protein was measured in human peritoneal dialysate samples with a sandwich ELISA using a previously described method with modifications ${ }^{18}$. Microtiter plates were coated overnight at $4{ }^{\circ} \mathrm{C}$ with a mouse monoclonal antibody (FibroGen, San Francisco, CA) that binds distinct epitopes in domain 2 of CTGF. Wells were rinsed and blocked with $1 \%$ bovine serum albumin overnight at $4{ }^{\circ} \mathrm{C}$. After washing, the $\mathrm{NH} 2$-terminal fragment of human recombinant CTGF (FibroGen), which was used for the calibration curve, and samples were added and incubated with alkaline phosphatase-conjugated antibody against CTGF (Leinco Technologies, Fenton, MO) overnight at $4^{\circ} \mathrm{C}$. Plates were washed, and substrate solution containing p-nitrophenyl phosphate was added. Absorbance was read at $405 \mathrm{~nm}$. 
The VEGF-C concentration in human PD effluents was measured with the Human VEGF-C Assay Kit (IBL, Takasaki, Japan) according to the manufacturer's instructions.

All samples for ELISA were evaluated in duplicate.

qPCR. Separate samples were collected for RNA extraction. Total RNA was extracted from tissue and cultured cells using RNeasy columns (Qiagen, Hilden, Germany). After cDNA synthesis, samples were mixed with TaqMan Gene Expression Assays (CTGF, Hs00170014_m1, Rn01537279_g1; VEGF-C, Hs00153458_m1, Rn00586458_m1, Mm00437310_m1; LYVE-1, Hs00272659_m1, Rn01510422_m1; podoplanin, Hs00366764_ m1, Rn00571195_m1; VEGFR-3, Mm01292604_m1; Applied Biosystems, Foster City, CA) that were run on Applied Biosystems Prism 7500HT. 18 S ribosomal RNA (4319413E) or TaqMan ${ }^{\circledR}$ Rodent GAPDH Control Reagents were used as an internal reference.

IHC and ISH. IHC for CTGF (sc-14939; Santa Cruz Biotechnology Inc., Dallas, TX), VEGF-C (Zymed Laboratories, South San Francisco, CA), D2-40 (BioLegend, San Diego, CA), LYVE-1 (Acris Antibodies GmbH, Herfold, Germany), and VEGFR-3 (R\&D Systems) was performed as previously reported ${ }^{13,34,53}$. To determine positive areas on stained sections, 10 random fields per section were chosen and photographed. LYVE-1-positive lymphatic vessels were identified and quantitated using Image J software, and the density was calculated for the analysis of lymphangiogenesis. CTGF and VEGF-C expression were semiquantitatively classified as follows: 0 , absent; 1 , mild; 2 , moderate; 3 , extensive ${ }^{13,54}$.

ISH to detect CTGF mRNA was performed on formalin-fixed paraffin-embedded rat diaphragm samples according to previously described methods ${ }^{18,55}$.

Statistical analysis. We expressed statistical values as the mean \pm standard deviation (SD). The Mann-Whitney U-test was used for the comparison between two independent groups. Variables of mRNA expression in human peritoneal biopsies were transformed into the logarithmic scale, and then a parametric test was performed. Ordinal CTGF and VEGF-C data in the CG model were compared using Spearman rank correlation. Pearson correlation was used for the other variables. All analyses were performed with SPSS software (SPSS, Chicago, IL). P values $<0.05$ were considered to be significant.

\section{Data Availability}

All data analyzed during this study are included in this published article and its Supplementary Information files.

\section{References}

1. Mactier, R. A. et al. Contribution of lymphatic absorption to loss of ultrafiltration and solute clearances in continuous ambulatory peritoneal dialysis. J Clin Invest 80, 1311-1316 (1987).

2. Abu-Hijleh, M. F., Habbal, O. A. \& Moqattash, S. T. The role of the diaphragm in lymphatic absorption from the peritoneal cavity. J Anat 186, 453-467 (1995).

3. Ates, K. et al. Effect of fluid and sodium removal on mortality in peritoneal dialysis patients. Kidney Int 60, 767-776 (2001).

4. Rumpsfeld, M., McDonald, S. P. \& Johnson, D. W. Higher peritoneal transport status is associated with higher mortality and technique failure in the Australian and New Zealand peritoneal dialysis patient populations. J Am Soc Nephrol 17, 271-278 (2006).

5. Brimble, K. S. et al. Meta-analysis: peritoneal membrane transport, mortality, and technique failure in peritoneal dialysis. J Am Soc Nephrol 17, 2591-2598 (2006).

6. Mizuno, M. et al. Recent analysis of status and outcomes of peritoneal dialysis in the Tokai area of Japan: the second report of the Tokai peritoneal dialysis registry. Clin Exp Nephrol 20, 960-971 (2016).

7. Williams, J. D. et al. Morphologic changes in the peritoneal membrane of patients with renal disease. J Am Soc Nephrol 13, 470-479 (2002).

8. Mateijsen, M. A. et al. Vascular and interstitial changes in the peritoneum of CAPD patients with peritoneal sclerosis. Perit Dial Int 19, 517-525 (1999).

9. Fusshöller, A., zur Nieden, S., Grabensee, B. \& Plum, J. Peritoneal fluid and solute transport: influence of treatment time, peritoneal dialysis modality, and peritonitis incidence. J Am Soc Nephrol 13, 1055-1060 (2002).

10. Parikova, A., Smit, W., Struijk, D. G. \& Krediet, R. T. Analysis of fluid transport pathways and their determinants in peritoneal dialysis patients with ultrafiltration failure. Kidney Int 70, 1988-1994 (2006).

11. Krediet, R. T. The effective lymphatic absorption rate is an accurate and useful concept in the physiology of peritoneal dialysis. Perit Dial Int 24, 309-313 (2004).

12. Flessner, M. Effective lymphatic absorption rate is not a useful or accurate term to use in the physiology of peritoneal dialysis. Perit Dial Int 24, 313-316 (2004).

13. Kinashi, H. et al. TGF- $\beta 1$ promotes lymphangiogenesis during peritoneal fibrosis. J Am Soc Nephrol 24, 1627-1642 (2013).

14. Zheng, W., Aspelund, A. \& Alitalo, K. Lymphangiogenic factors, mechanisms, and applications. J Clin Invest 124, 878-887 (2014).

15. Coso, S., Bovay, E. \& Petrova, T. V. Pressing the right buttons: signaling in lymphangiogenesis. Blood 123, 2614-2624 (2014).

16. Kinashi, H. et al. Roles of the TGF- $\beta^{-}$VEGF-C Pathway in Fibrosis-Related Lymphangiogenesis. Int J Mol Sci 19, E2487, https://doi. org/10.3390/ijms19092487 (2018).

17. Terabayashi, T. et al. Vascular endothelial growth factor receptor-3 is a novel target to improve net ultrafiltration in methylglyoxalinduced peritoneal injury. Lab Invest 95, 1029-1043 (2015).

18. Mizutani, M. et al. Connective tissue growth factor (CTGF/CCN2) is increased in peritoneal dialysis patients with high peritoneal solute transport rate. Am J Physiol Renal Physiol 298, F721-733 (2010).

19. Zarrinkalam, K. H. et al. Connective tissue growth factor and its regulation in the peritoneal cavity of peritoneal dialysis patients. Kidney Int 64, 331-338 (2003).

20. Leung, J. C. et al. Regulation of CCN2/CTGF and related cytokines in cultured peritoneal cells under conditions simulating peritoneal dialysis. Nephrol Dial Transplant 24, 458-469 (2009).

21. Pi, L. et al. CCN2/CTGF regulates neovessel formation via targeting structurally conserved cystine knot motifs in multiple angiogenic regulators. FASEB J 26, 3365-3379 (2012).

22. Kinashi, H. et al. Connective tissue growth factor regulates fibrosis-associated renal lymphangiogenesis. Kidney Int $\mathbf{9 2 , 8 5 0 - 8 6 3}$ (2017).

23. Huang, J. L. et al. Vascular Endothelial Growth Factor C for Polycystic Kidney Diseases. J Am Soc Nephrol 27, 69-77 (2016).

24. Hasegawa, S. et al. Vascular endothelial growth factor-C ameliorates renal interstitial fibrosis through lymphangiogenesis in mouse unilateral ureteral obstruction. Lab Invest 97, 1439-1452 (2017). 
25. Hwang, S. D. et al. Inhibition of lymphatic proliferation by the selective VEGFR-3 inhibitor SAR131675 ameliorates diabetic nephropathy in db/db mice. Cell Death Dis 10, 219, https://doi.org/10.1038/s41419-019-1436-1 (2019).

26. Ueno, T. et al. Mesenchymal stem cells ameliorate experimental peritoneal fibrosis by suppressing inflammation and inhibiting TGF- $\beta 1$ signaling. Kidney Int 84, 297-307 (2013).

27. Toda, N. et al. Crucial Role of Mesangial Cell-derived Connective Tissue Growth Factor in a Mouse Model of Anti-Glomerular Basement Membrane Glomerulonephritis. Sci Rep 7, 42114, https://doi.org/10.1038/srep42114 (2017).

28. Toda, N. et al. Deletion of connective tissue growth factor ameliorates peritoneal fibrosis by inhibiting angiogenesis and inflammation. Nephrol Dial Transplant 33, 943-954 (2018).

29. Smit, W. et al. Analysis of the prevalence and causes of ultrafiltration failure during long-term peritoneal dialysis: a cross-sectional study. Perit Dial Int 24, 562-570 (2004).

30. Alitalo, A. \& Detmar, M. Interaction of tumor cells and lymphatic vessels in cancer progression. Oncogene 31, 4499-4508 (2012).

31. Kim, H., Kataru, R. P. \& Koh, G. Y. Inflammation-associated lymphangiogenesis: a double-edged sword? J Clin Invest 124, $936-942$ (2014).

32. Dashkevich, A. et al. VEGF Pathways in the Lymphatics of Healthy and Diseased Heart. Microcirculation 23, 5-14 (2016)

33. Cui, Y. et al. Therapeutic lymphangiogenesis ameliorates established acute lung allograft rejection. J Clin Invest 125, 4255-4268 (2015).

34. Sakamoto, I. et al. Lymphatic vessels develop during tubulointerstitial fibrosis. Kidney Int 75, 828-838 (2009).

35. Vlahu, C. A. et al. Lymphangiogenesis and Lymphatic Absorption Are Related and Increased in Chronic Kidney Failure, Independent of Exposure to Dialysis Solutions. Adv Perit Dial 31, 21-25 (2015).

36. Davies, S. J. Unraveling the mechanisms of progressive peritoneal membrane fibrosis. Kidney Int 89, 1185-1187 (2016).

37. Yang, W. S. et al. Intraperitoneal vascular endothelial growth factor $\mathrm{C}$ level is related to peritoneal dialysis ultrafiltration. Blood Purif 28, 69-74 (2009)

38. Gerritsen, K. G. et al. Effect of GFR on plasma N-terminal connective tissue growth factor (CTGF) concentrations. Am J Kidney Dis 59, 619-627 (2012)

39. Yu, Z., Lambie, M. \& Davies, S. J. Longitudinal study of small solute transport and peritoneal protein clearance in peritoneal dialysis patients. Clin I Am Soc Nephrol 9, 326-334 (2014).

40. Rippe, B. \& Davies, S. Permeability of peritoneal and glomerular capillaries: what are the differences according to pore theory? Perit Dial Int 31, 249-258 (2011).

41. Yung, S. \& Chan, T. M. Intrinsic cells: mesothelial cells-central players in regulating inflammation and resolution. Perit Dial Int 29, S21-27 (2009).

42. Kang, D. H. et al. High glucose solution and spent dialysate stimulate the synthesis of transforming growth factor-betal of human peritoneal mesothelial cells: effect of cytokine costimulation. Perit Dial Int 19, 221-230 (1999).

43. De Vriese, A. S., Tilton, R. G., Mortier, S. \& Lameire, N. H. Myofibroblast transdifferentiation of mesothelial cells is mediated by RAGE and contributes to peritoneal fibrosis in uraemia. Nephrol Dial Transplant 21, 2549-2555 (2006)

44. Lai, K. N. et al. Changes of cytokine profiles during peritonitis in patients on continuous ambulatory peritoneal dialysis. Am J Kidney Dis 35, 644-652 (2000).

45. Yokoi, H. et al. Reduction in connective tissue growth factor by antisense treatment ameliorates renal tubulointerstitial fibrosis. J Am Soc Nephrol 15, 1430-1440 (2004)

46. Guha, M. et al. Specific down-regulation of connective tissue growth factor attenuates progression of nephropathy in mouse models of type 1 and type 2 diabetes. FASEB J 21, 3355-3368 (2007).

47. Luo, G. H. et al. Inhibition of connective tissue growth factor by small interfering RNA prevents renal fibrosis in rats undergoing chronic allograft nephropathy. Transplant Proc 40, 2365-2369 (2008).

48. Okada, H. et al. Connective tissue growth factor expressed in tubular epithelium plays a pivotal role in renal fibrogenesis. J Am Soc Nephrol 16, 133-143 (2005).

49. Sakai, N. et al. Inhibition of CTGF ameliorates peritoneal fibrosis through suppression of fibroblast and myofibroblast accumulation and angiogenesis. Sci Rep 7, 5392, 1038/s41598-017-05624-2 (2017).

50. Suzuki, Y. et al. Transforming growth factor- $\beta$ induces vascular endothelial growth factor-C expression leading to lymphangiogenesis in rat unilateral ureteral obstruction. Kidney Int 81, 865-879 (2012).

51. Honda, K. et al. Impact of uremia, diabetes, and peritoneal dialysis itself on the pathogenesis of peritoneal sclerosis: a quantitative study of peritoneal membrane morphology. Clin J Am Soc Nephrol 3, 720-728 (2008).

52. Yokoi, H. et al. Pleiotrophin triggers inflammation and increased peritoneal permeability leading to peritoneal fibrosis. Kidney Int 81, 160-169 (2012)

53. Falke, L. L. et al. Age-dependent shifts in renal response to injury relate to altered BMP6/CTGF expression and signaling. Am J Physiol Renal Physiol 311, F926-934 (2016).

54. Kato, H. et al. Atrial natriuretic peptide ameliorates peritoneal fibrosis in rat peritonitis model. Nephrol Dial Transplant 27, 526-536 (2012).

55. Ito, Y. et al. Expression of connective tissue growth factor in human renal fibrosis. Kidney Int 53, 853-861 (1998).

\section{Acknowledgements}

We are grateful for the technical assistance of Ms. Naoko Asano, Ms. Yuriko Sawa, Mr. Norihiko Suzuki, Ms. Keiko Higashide (Department of Nephrology, Nagoya University, Nagoya, Japan), Ms. Mai Yamauchi (Department of Nephrology and Rheumatology, Aichi Medical University, Nagakute, Japan), and Mr. Roel Broekhuizen (Department of Pathology, University Medical Center Utrecht, Utrecht, The Netherlands). This work was supported by a JSPS Grant-in-Aid for Research Activity Start-up Grant Number JP17H06746 and the Japanese Association of Dialysis Physicians.

\section{Author Contributions}

H.K., T.N., R.G. and Y.I. designed the study. Y.S., T.K., M.M. and S.M. contributed to sample preparation. N.T., H.Y. and M.Y. planned and performed the mouse experiments. H.K., N.T., T.S. and J.A. carried out the experiments. H.K. wrote the manuscript with support from T.N. and R.G.

Additional Information

Supplementary information accompanies this paper at https://doi.org/10.1038/s41598-019-48699-9.

Competing Interests: R. Goldshcmeding, co-author of this manuscript, received a grant from FibroGen Inc., which develops an anti-CTGF drug. CTGF is a main subject of this manuscript.

Publisher's note: Springer Nature remains neutral with regard to jurisdictional claims in published maps and institutional affiliations. 
(c) (i) Open Access This article is licensed under a Creative Commons Attribution 4.0 International License, which permits use, sharing, adaptation, distribution and reproduction in any medium or format, as long as you give appropriate credit to the original author(s) and the source, provide a link to the Creative Commons license, and indicate if changes were made. The images or other third party material in this article are included in the article's Creative Commons license, unless indicated otherwise in a credit line to the material. If material is not included in the article's Creative Commons license and your intended use is not permitted by statutory regulation or exceeds the permitted use, you will need to obtain permission directly from the copyright holder. To view a copy of this license, visit http://creativecommons.org/licenses/by/4.0/.

(C) The Author(s) 2019 